\title{
Adaptación del Inventario de la Triada Cognitiva Infantil en población española
}

\author{
Luis M. Lozano ${ }^{1}$, Inmaculada Valor-Segura ${ }^{1}$, Ignacio Pedrosa ${ }^{2}$, Javier Suárez-Álvarez², \\ Eduardo García-Cueto ${ }^{2}$ y Luis Lozano ${ }^{3}$ \\ 1 Universidad de Granada (España). \\ 2Universidad de Oviedo (España). \\ 3 Consejería de Educación y Cultura, Gobierno del Principado de Asturias (España).
}

\begin{abstract}
Resumen: La depresión es uno de los trastornos mentales que más preocupan a nivel mundial. Una de las teorías que más investigación ha suscitado es la teoría cognitiva de la depresión de Beck. El Inventario de Tríada Cognitiva (CTI) se ha elaborado para evaluar los diferentes componentes de la tríada cognitiva, si bien la mayoría de sus estudios se han desarrollado en población adulta y adolescente. El objetivo de este trabajo es mostrar evidencias de validez factorial de la versión reducida del CTI, así como el estudio de las propiedades psicométricas del cuestionario en población infantil. En una muestra de 3292 niños de edades entre los 8 y los 13 años $(M=9.8 ; D T=1.24)$, los resultados mostraron que el mejor ajuste factorial se obtiene con una estructura bifactorial (tríada positiva y tríada negativa). La fiabilidad de las diferentes dimensiones es buena $(\alpha=.88$ y .92 , respectivamente) y las evidencias de validez convergente adecuadas. El cuestionario fue evaluado también desde la perspectiva de la Teoría de la Respuesta a los Ítems mostrando unas propiedades correctas. Finalmente se realizó la baremación del cuestionario atendiendo a las diferencias por género encontradas previamente.
\end{abstract}

Palabras clave: Depresión; triada cognitiva; fiabilidad; validez.

\section{Introducción}

La depresión es, actualmente, uno de los trastornos mentales que más preocupa a nivel mundial, dada su prevalencia, sus efectos en la vida de las personas y el alto coste económico que conlleva (Bernaras, Jaureguizar, Soroa, Ibabe y De las Cuevas, 2013; Dobson y Dozois, 2008).

Una de las teorías cognitivas que más investigación ha suscitado es la teoría cognitiva de la depresión de Beck (1967, 1983). Un elemento central de esta teoría es el constructo de esquema. Se considera que las personas que poseen un esquema de pensamiento depresivo, ante la aparición en su vida de un suceso aversivo, presentan una mayor probabilidad de procesar la información autorreferente de una manera negativa. Es decir, que la persona deprimida se caracterizaría por procesar la información emocional autorreferente de una manera sesgada, al atender especialmente a la información negativa e ignorando otro tipo de información presente, principalmente aquella que es positiva (Beck, 1991; Sánchez y Vázquez, 2012).

Estos sesgos cognitivos favorecen la aparición de pensamientos negativos sobre sí mismo, el mundo y el futuro. Por lo tanto, las personas deprimidas tienden a atribuir sus experiencias desagradables a un defecto personal, de tipo psicológico, moral o físico; entienden que el mundo les hace demandas exageradas y/o les presenta obstáculos insuperables para alcanzar sus objetivos; y anticipan que sus dificul-

* Dirección para correspondencia [Correspondence address]: Eduardo García-Cueto. Universidad de Oviedo. Facultad de Psicología. Plaza Feijóo, s/n. Despacho 214. CP 33003. Oviedo (España). E-mail: cueto@uniovi.es
Title: Adapting the Cognitive Triad Inventory for Children Spanish population.

Abstract: Depression is one of the mental disorders that concern worldwide. One theory that has attracted more researches is the Beck's cognitive theory of depression. The Cognitive Triad Inventory (CTI) has been developed to evaluate the different components of the cognitive triad, although most studies had been developed in adult and adolescent population. The aim of this work is to show evidence of factorial validity of the reduced version of the CTI, and the study of the psychometric properties of the inventory in children. In the sample of 3292 children with age between 8 and $13(M=9.86 ; S D=1.24)$, the results showed a better factorial fit with a bifactorial model (positive triad and negative triad). The reliability of the dimensions is good ( $\alpha=.88$ and .92 respectively) and convergent validity evidence is adequate. The inventory was evaluated from the Item Response Theory framework, showing correct properties. Finally the norms of the questionnaire were made addressing the gender differences that were found previously.

Key words: Depression; cognitive triad; reliability; validity.

tades y sufrimientos actuales continuarán indefinidamente (Beck, Rush, Shaw y Emery, 1979). Estos tres grupos de juicios negativos son denominados Tríada Cognitiva negativa de la depresión, la cual constituye otro principio central de esta teoría, al considerarse responsable del mantenimiento y exacerbación del resto de síntomas depresivos (Beck, 1987).

La investigación existente sobre la teoría cognitiva de la depresión de Beck ha desarrollado la mayoría de sus estudios en población adulta y adolescente (e.g. Estévez y Calvete, 2009; Morris, Ciesla y Garber, 2008). Dado que es una teoría de diáthesis-estrés, la investigación se ha centrado en dos aspectos fundamentales: por una parte, se han tratado de confirmar los componentes presentes en la relación entre la diáthesis (esquemas cognitivos vulnerables, depresivos) y el estrés ocasionado por situaciones vitales negativas, aversivas (Auerbach, Eberhart y Abela, 2010). Por otro lado, se ha profundizado en las relaciones que la tríada cognitiva presenta con diferentes trastornos psicológicos como la depresión (Beshai, 2010), la ansiedad (Shin, 2008) o la ideación suicida (Chang, Lin, y Lin 2007; Reinherz, Tanner, Berger, Bearsdslee y Fitzmaurice, 2006), entre otros.

A la hora de llevar a cabo la evaluación de los diferentes componentes de la tríada cognitiva, uno de los instrumentos de medida diseñados para tal fin es el Inventario de Tríada Cognitiva (CTI; Beckham, Leber, Watkins, Boyer y Cook, 1986).

Aunque originariamente este instrumento se planteó bajo una estructura tridimensional, diversos estudios en que se ha analizado la estructura factorial han dado cuenta de la inexistencia de acuerdo respecto a su dimensionalidad, refutando la distinción conceptual de los tres componentes de la tríada. Así, McIntosh y Fisher (2000) definieron un único factor 
que denominaron Actitud negativa auto-relevante. Igualmente, Haaga, Dyck y Ernst (1991) obtuvieron un único factor relacionado con el yo, y, por otro lado, Anderson y Skidmore (1995) cinco factores: yo positivo, mundo negativo, mundo positivo, futuro positivo y futuro negativo.

Ante la falta de instrumentos que permitiesen evaluar la tríada cognitiva planteada por Beck en población joven, Kaslow, Stark, Printz, Livingston y Tsai (1992) realizaron una revisión del CTI con objeto de desarrollar un instrumento que cubriese esta necesidad. Así, elaboraron el Inventario de la Tríada Cognitiva para Niños (CTI-C; Kaslow et al., 1992). Sin embargo, al igual que sucede en los estudios realizados con adultos, los resultados derivados de esta versión tampoco ha sido consistente en la diferenciación de los tres componentes que Beck $(1967,1983)$ propone teóricamente. Así, algunos estudios han obtenido tres factores, aunque éstos no concuerdan con los defendidos por Beck (Greening, Stoppelbein, Dhossche y Martin, 2005; Zauszniewski, Panitrat y Youngblut, 1999), mientras que LaGrange et al. (2008) han mostrado dos factores unipolares. El primero formado únicamente por pensamientos positivos sobre el yo, mundo y futuro y el segundo por los pensamientos negativos.

Considerando, tal y como se dijo anteriormente, que la tríada cognitiva presenta una implicación directa en el desarrollo y mantenimiento de la depresión, contar con un instrumento en castellano que informe sobre estos aspectos y esté adecuadamente validado en población infantil resulta fundamental para el trabajo preventivo y clínico con niños. Por este motivo, en el presente trabajo se propone como objetivo principal aportar evidencias de validez factorial de la versión breve del CTI-C, comprobando la estructura tridimensional de la tríada cognitiva en niños, tal y como se defiende en la teoría cognitiva de la depresión de Beck (1967, 1983). Además, se presenta una versión abreviada del CTI-C adaptada a la población general infantil española a partir de la cual se aportan sus propiedades psicométricas. Estos datos se combinan con un abordaje de las propiedades del test desde la Teoría de Respuesta al Ítem, lo cual, resultando novedoso, complementa la información dada sobre esta escala bajo el modelo de la Teoría Clásica de los Tests en estudios precedentes. Finalmente, tras el estudio de las diferencias en función del sexo, se aportan baremos adaptados a esta población que permiten la aplicación e interpretación del instrumento en el ámbito aplicado.

\section{Método}

\section{Participantes}

La muestra estuvo compuesta por 3292 niños de edades comprendidas entre los 8 y los 13 años $(M=9.86$; $D T=1.24)$, escolarizados entre $3^{\text {er }}$ y $6^{\circ}$ curso de Educación Primaria (aproximadamente un $25 \%$ por cada uno de los cuatro cursos mencionados). El 51.3\% fueron niños y el $48.7 \%$ niñas. La muestra pertenece al Principado de Asturias. El muestreo fue aleatorio estratificado por zonas: rural (interior y costa) y urbana. También por tipo de centro: público, privado y concertado. Con los datos obtenidos y este tamaño muestral, el error máximo cometido en las estimaciones, al nivel de confianza del $95 \%$, es de \pm 0.48 .

\section{Instrumentos}

Tras la obtención de las preceptivas autorizaciones se aplicaron los siguientes instrumentos de evaluación.

Inventario de la Tríada Cognitiva para Niños (CTI-C; Kaslow et al., 1992). Es una prueba de 36 ítems que evalúa la visión que la persona tiene de sí mismo, del mundo y del futuro. Cada subescala incluye 12 ítems, la mitad redactados en forma positiva y la otra mitad en forma negativa a los que hay que contestar con tres formas alternativas: "sî́, "quizás" y "no". Los autores informaron de un coeficiente alfa para la prueba total de .92 . En cuanto a sus subescalas, su fiabilidad fue de .83 para el Yo, .69 para el Mundo y .85 para el Futuro. La adaptación al castellano se realizó mediante un proceso de retro-traducción siguiendo las directrices internacionales para la traducción y adaptación de pruebas psicométricas (Muñiz, Elosua y Hambleton, 2013). Ambas traducciones, tanto la directa como la inversa, fueron realizadas por traductores profesionales independientes cuya lengua materna es el inglés. Para resolver el reducido número de casos en los que se constató alguna inconsistencia, se reunió a ambos traductores con el grupo de investigación y se llegó a un consenso sobre la traducción más adecuada.

Sobre esta escala se realizaron algunos cambios. En primer lugar se convirtió el formato de respuesta en una escala tipo Likert de 5 alternativas, pues las medidas obtenidas presentan claras ventajas cuando se utiliza este tipo de escala (Lozano, García-Cueto y Muñiz, 2008; Muñiz, García-Cueto y Lozano, 2005). Dado que el instrumento ha sido diseñado para niños, las respuestas no se plantearon como una decisión numérica. Simplemente debían indicar la frecuencia con la que les ocurrían las afirmaciones presentadas, atendiendo a las etiquetas verbales mostradas en el cuestionario. Durante todo el proceso el psicólogo que aplicó la prueba estuvo atento para resolver cualquier tipo de duda en la comprensión de las instrucciones del inventario, de los ítems o de la forma de responder. Por otro lado, con la finalidad de contar con un instrumento más breve, se eliminaron los 18 ítems que mostraron peores propiedades psicométricas, tal y como se explica en la sección de Resultados. Así, se definió un cuestionario de 18 ítems denominado CTI-C(18).

La escala de Autovaloración del IPI $(\alpha=.89)$. Esta escala, compuesta por nueve ítems, evalúa la presencia de juicios negativos cuando su ejecución no es todo lo excelente que desea (Lozano, García-Cueto, Martín y Lozano, 2012). Cuanto mayor es la puntuación peor es la autovaloración que el niño realiza de sí mismo.

Las siguientes cuatro escalas del Cuestionario Educativo Clínico: Ansiedad y Depresión (CECAD; Lozano, GarcíaCueto y Lozano, 2010): 
Inutilidad $(\alpha=.91)$. Está formada por nueve ítems que describen la percepción sobre la valía personal en cuanto a la capacidad para enfrentarse a las tareas cotidianas.

Irritabilidad $(\alpha=.87)$. Está formada por siete ítems, en donde cuanta más alta sea la puntuación, la persona se percibe con una gran facilidad para enfadarse y con sensación interna de rabia.

Depresión $(\alpha=.95)$. Está formada por 29 ítems. Una elevada puntuación indica un estado de ánimo depresivo o irritable, insomnio o hipersomnia, inutilidad, pérdida de energía, disminución de la capacidad de pensar, y pensamiento suicida.

Ansiedad $(\alpha=.91)$. Consta de 19 ítems, y en ella las puntuaciones elevadas son sintomáticas de tensión muscular y problemas inherentes a una incorrecta respiración, miedos, preocupaciones y pensamientos molestos.

Todos los cuestionarios anteriores se contestaban con un formato de respuesta en una escala tipo Likert de 5 alternativas.

\section{Procedimiento}

Las pruebas fueron aplicadas por psicólogos expertos en la administración de tests. La evaluación se realizó de manera colectiva en las aulas proporcionadas por cada centro educativo. La participación fue totalmente voluntaria, sin ninguna recompensa y bajo los requisitos exigidos por el Comité de Ética en Investigación.

\section{Análisis de datos}

Se utilizó el estadístico de Levene para comprobar la homocedasticidad y la prueba de Shapiro-Wilk para comprobar la normalidad de las distribuciones.

Para probar si los datos se ajustaban mejor a una estructura bidimensional o tridimensional se llevaron a cabo sendos análisis factoriales confirmatorios, extrayendo dos y tres factores, respectivamente. Se empleó el método de extracción de Máxima Verosimilitud Robusta, por no cumplir los datos los supuestos de normalidad y homocedasticidad. Además, se calcularon los pesos factoriales de cada ítem en el factor.

Con la finalidad de detectar posibles comportamientos extraños de los ítems que pudieran afectar a la validez del test, se llevó a cabo un estudio del funcionamiento diferencial de los ítems (DIF) tanto en función del sexo como del ciclo educativo (segundo y tercer ciclo de Educación Primaria). Para ello se utilizó el método de regresión logística, teniendo en cuenta tanto el test de significación estadística como el tamaño del efecto obtenido con objeto de reducir la tasa de posibles falsos positivos $\left(p<.01 ; R^{2}>.035\right.$; GomezBenito, Hidalgo y Zumbo, 2013).

El índice de discriminación de los ítems se calculó mediante correlación parcial eliminando el influjo del ítem en el test (Muñiz, Fidalgo, García-Cueto, Martínez y Moreno, 2005).
Las evidencias de validez convergente, se estimaron mediante el coeficiente de correlación de Pearson y aplicando sobre éste la corrección de los errores de atenuación.

La fiabilidad de los tests se estimó mediante el coeficiente alfa para escalas ordinales (Elosua y Zumbo, 2008).

Para el estudio de las diferencias en función del sexo se utilizó la prueba U de Mann-Whitney y para las diferencias entre cursos el test de Kruskal-Wallis $(\mathrm{NC}=95 \%)$. En ambos acasos dicho estudio se complementó con el cálculo del tamaño del efecto mediante los métodos delta de Cliff (1993) y eta-cuadrado (Morse, 1999).

Desde la perspectiva de la Teoría de la Respuesta a los Ítems, se estimaron los parámetros de posicionamiento $(b)$ y discriminación (a) de cada una de las escalas, así como su función de información bajo el Modelo de Respuesta Graduada de Samejima (2004). Este modelo es el adecuado para analizar desde esta perspectiva los ítems de una escala tipo Likert. Con este modelo se obtiene la discriminación de cada ítem (parámetro a) y tantos parámetros de localización como número de alternativas menos uno (parámetros b). Los parámetros $b$ indican la probabilidad condicionada de que una persona obtenga una determinada puntuación en el ítem a partir de la diferencia entre el valor que van a señalar y el superior.

Finalmente, los resultados del test fueron baremados en una escala de 13 puntos centiles.

Todos los análisis estadísticos se llevaron a cabo con el SPSS 20, FACTOR 9.2, Multilog 7.03 y Mplus 5.1.

\section{Resultados}

En primer lugar se comprobaron tanto el supuesto de igualdad de varianzas como el de normalidad. Partiendo de la hipótesis de bidimensionalidad, los resultados obtenidos con el CTI-C(18) no permiten defender la igualdad de varianzas en la población en función del sexo en ninguna de las dimensiones $\left(p_{T N}=.008, p_{T P}<.001\right)$. En función del curso, sí se puede defender la homocedasticidad en la dimensión positiva $\left(p_{T P}=.724\right)$, pero no en la negativa $\left(p_{T N}=.003\right)$. Asimismo, la prueba de Shapiro-Wilk determinó que ninguna de las dos dimensiones se ajusta a una distribución normal (en ambos casos $p<.001)$.

Habida cuenta de la falta de acuerdo en las diversas investigaciones sobre la dimensionalidad del test en niños (Kaslow et al., 1992; LaGrange et al., 2008; Zauszniewski et al., 1999), se sometió a prueba, mediante un Análisis Factorial Confirmatorio, la estructura trifactorial (yo, mundo y futuro) obteniendo los índices de ajuste que se exponen en la Tabla 1.

Puesto que el ajuste obtenido fue deficiente se comprobó el ajuste de los datos a un modelo bidimensional, tal y como proponen LaGrange et al. (2008). Los índices de ajuste y la información aportada por el modelo se pueden observar en la Tabla 1. 
Tabla 1. Análisis de la estructura factorial del CTI-C(18)

\begin{tabular}{lllllll}
\hline Estructura & $\chi^{2} / \mathrm{gl}$ & RMSEA & I.C. RMSEA $(90 \%)$ & SRMR & CFI & AIC \\
\hline Tridimensional & 21.38 & .088 & {$[.085-.091]$} & .088 & .741 & 131056.775 \\
Bidimensional & 5.59 & .042 & {$[.039-.045]$} & .037 & .940 & 128110.975 \\
\hline
\end{tabular}

Nota: RMSEA=Raíz cuadrada media del error típico de aproximación; I.C.=Intervalo Confidencial; SRMR: Raíz cuadrada de los residuales estandarizados; $\mathrm{CFI}=$ Índice de ajuste comparativo; $\mathrm{AIC}=$ Criterio de Información de Akaike.

Una vez confirmada la estructura bidimensional del instrumento se decidió elaborar la versión breve del cuestionario para población infantil. Así, sobre la muestra total y bajo la propuesta bidimensional, se estudió el posible funcionamiento diferencial de los ítems para, posteriormente, calcular tanto el índice de discriminación de los ítems como su peso factorial. En primer lugar, ninguno de los ítems del instrumento mostró funcionamiento diferencial ni en función del sexo ni del ciclo educativo. Tras comprobar la inexistencia de DIF, a partir de la escala original, se decidió eliminar aquellos ítems con índices de discriminación o pesos factoriales inferiores a .20 (Muñiz et al., 2005). Bajo este criterio se eliminaron 18 ítems, quedando el cuestionario definitivo constituido por otros 18, nueve de ellos en la dimensión negativa y nueve en la positiva (Tabla 2). Los descriptivos de la escala breve obtenida se muestran en la Tabla 3.

Para aportar evidencias de validez convergente se calculó la correlación entre los factores negativo y positivo del CTIC(18) las dimensiones de Depresión, Ansiedad, Irritabilidad, Inutilidad y Autovaloración del CECAD. Las correlaciones fueron corregidas mediante las fórmulas de atenuación (Tabla 4). Como se observa, las mayores correlaciones se obtuvieron, en ambos casos, respecto a la dimensión de depresión.

Tabla 3. Estadísticos descriptivos de la TCT para el CTI-C(18).

\begin{tabular}{|c|c|c|c|c|c|c|c|c|}
\hline \multirow[b]{3}{*}{ Estadísticos descriptivos } & \multicolumn{4}{|c|}{ Hombres } & \multicolumn{4}{|c|}{ Mujeres } \\
\hline & \multicolumn{2}{|c|}{ Rural } & \multicolumn{2}{|c|}{ Urbano } & \multicolumn{2}{|c|}{ Rural } & \multicolumn{2}{|c|}{ Urbano } \\
\hline & $\begin{array}{l}2^{\circ} \text { ciclo } \\
(\mathrm{n}=163)\end{array}$ & $\begin{array}{l}\text { 3er ciclo } \\
(\mathrm{n}=189)\end{array}$ & $\begin{array}{l}2^{\circ} \text { ciclo } \\
(n=677)\end{array}$ & $\begin{array}{l}3^{\mathrm{er}} \text { ciclo } \\
(\mathrm{n}=660)\end{array}$ & $\begin{array}{l}2^{\circ} \text { ciclo } \\
(n=183)\end{array}$ & $\begin{array}{l}\text { 3er ciclo } \\
(\mathrm{n}=192)\end{array}$ & $\begin{array}{l}2^{\circ} \text { ciclo } \\
(n=606)\end{array}$ & $\begin{array}{l}3^{\text {er }} \text { ciclo } \\
(\mathrm{n}=622)\end{array}$ \\
\hline$r_{\text {ix Tríada - }}$ & $.33-.74$ & $.23-.69$ & $.34-.64$ & $.37-.70$ & $.22-.73$ & $.25-.68$ & $.28-.62$ & $.30-.67$ \\
\hline$\alpha_{\text {Tríada - }}$ & .85 & .83 & .83 & .85 & .81 & .82 & .81 & .83 \\
\hline Media Tríada - & 19.23 & 18.27 & 18.30 & 17.77 & 18.49 & 17.09 & 16.96 & 16.61 \\
\hline DT Tríada - & 7.75 & 6.33 & 7.14 & 6.73 & 6.44 & 5.99 & 6.47 & 6.11 \\
\hline$r_{\mathrm{x}}$ Tríada + & $.30-.62$ & $.45-.63$ & $.43-.57$ & $.51-.60$ & $.31-.62$ & $.53-.57$ & $.34-.51$ & $.46-.66$ \\
\hline$\alpha_{\text {Tríada }}+$ & .80 & .84 & .82 & .85 & .78 & .83 & .77 & .84 \\
\hline Media Tríada+ & 36.52 & 35.53 & 36.28 & 36.68 & 37.27 & 37.75 & 38.12 & 38.07 \\
\hline$\underline{D T}_{\text {Tríada }}+$ & 6.16 & 6.14 & 6.72 & 6.36 & 5.63 & 5.41 & 5.70 & 5.59 \\
\hline
\end{tabular}

Nota: $r_{i x}=$ indice de discriminación; $D T=$ Desviación típica; Tríada $-=$ Tríada negativa; Tríada $+=$ Tríada positiva.

Tabla 4. Matriz de correlaciones entre el CTI-C(18) y las dimensiones del CECAD.

\begin{tabular}{lcccc}
\hline & \multicolumn{2}{c}{ Tríada Negativa } & \multicolumn{2}{c}{ Tríada Positiva } \\
\hline & $\mathrm{r}_{\mathrm{xy}}$ & $\mathrm{r}_{\mathrm{xy}}^{\prime}$ & $\mathrm{r}_{\mathrm{xy}}$ & $\mathrm{r}_{\mathrm{xy}}^{\prime}$ \\
\hline Inutilidad & .67 & .73 & -.35 & -.40 \\
Irritabilidad & .55 & .62 & -.28 & -.33 \\
Autovaloración & .63 & .70 & -.25 & -.28 \\
Depresión & .73 & .79 & -.37 & -.41 \\
Ansiedad & .59 & .65 & -.26 & -.29 \\
\hline
\end{tabular}

Nota: $r_{x y}=$ Coeficiente de correlación de Pearson; $r_{x y}^{\prime}=$ Coeficiente de atenuación.
Tabla 2. Propiedades psicométricas del CTI-C(18).

\begin{tabular}{llll}
\hline \multicolumn{3}{c}{ Factores } \\
Ítems & TN & TP & ID \\
\hline 1 & .73 & & .63 \\
2 & .77 & & .65 \\
3 & .31 & & .31 \\
4 & .71 & & .65 \\
5 & .72 & & .61 \\
6 & .65 & & .60 \\
7 & .65 & & .56 \\
8 & .64 & & .59 \\
9 & .35 & & .35 \\
10 & & .56 & .50 \\
11 & & .60 & .53 \\
12 & & .61 & .56 \\
13 & & .58 & .52 \\
14 & & .61 & .54 \\
15 & & .66 & .55 \\
16 & & .62 & .55 \\
17 & & .57 & .51 \\
18 & & .56 & .49 \\
\hline Coeficiente alfa ordinal & .92 & .88 & \\
\hline Nota: TN=Fat de Tra & & & \\
\hline
\end{tabular}

Nota: $\mathrm{TN}=$ Factor de la Tríada Negativa; $\mathrm{TP}=$ Factor de la Tríada positiva; ID=Índices de discriminación de los ítems.
En la Tabla 5 se muestran las diferencias encontradas en función del sexo, siendo éstas estadísticamente significativas en ambas dimensiones y obteniendo los hombres puntuaciones más elevadas en ambos factores. En cuanto al estudio de las diferencias en función del curso, éstas no fueron estadísticamente significativas en ninguno de los dos factores. En ambos acasos dicho estudio se complementó con el cálculo del tamaño del efecto (Tabla 5). 
Tabla 5. Diferencias en función del sexo y el curso en el CTI-C(18).

\begin{tabular}{lccccc}
\hline & \multicolumn{3}{c}{ Sexo } & \multicolumn{3}{c}{ Curso } \\
\hline $\mathrm{U}$ & $\mathrm{TN}$ & $\mathrm{TP}$ & & $\mathrm{TN}$ & $\mathrm{TP}$ \\
$\mathrm{z}$ & 815,960 & 798,264 & $\chi^{2}$ & 5.24 & 1.29 \\
$p$ & -4.377 & $-5,258$ & $\mathrm{~g}$ & 3 & 3 \\
$\delta$ & $<.001$ & $<.001$ & $p$ & .155 & .732 \\
\hline & .12 & .10 & $\eta^{2}$ & .002 & $<.001$ \\
\hline
\end{tabular}

Nota: U=Estadístico de Mann-Whitney; z=Puntuación estandarizada de distribución normal; $\mathrm{TN}=$ Factor de la Tríada Negativa; $\mathrm{TP}=$ Factor de la Tríada positiva; $\delta=$ Tamaño del efecto (Delta de Cliff); $\eta^{2}=$ Tamaño del efecto (eta cuadrado).

Con el fin de analizar la precisión en la medida de la escala en función de las puntuaciones de los participantes, se analizaron las Funciones de Información de ambas dimensiones del test (Figura 1). En ellas, la línea continua representa la función de información y la puntuada el error típico de medida. Como puede observarse, en ambas subescalas existe un amplio abanico de puntuaciones $\theta$ con una estimación muy precisa, especialmente para las puntuaciones medias altas en la tríada negativa y medias bajas para la positiva. Esto indica que aporta información más precisa en las puntuaciones que permiten detectar posibles casos problemáticos.

En la Tabla 6 se presenta la estimación de los parámetros de discriminación y posicionamiento para cada uno de los ítems del test, estimados mediante el Modelo de Respuesta Graduada de Samejima. La columna $b_{1}-b_{4}$ indica la facilidad para conseguir un cambio de actitud desde lo más negativo del ítem hacia lo más positivo. Esta información puede resultar especialmente útil para orientar la intervención psicológica.

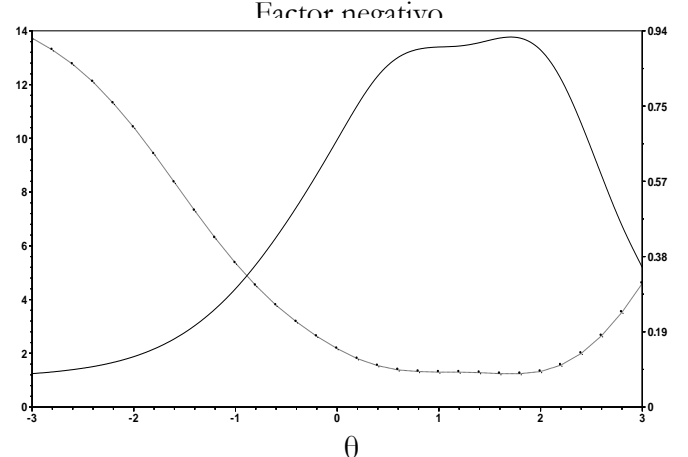

$\theta$

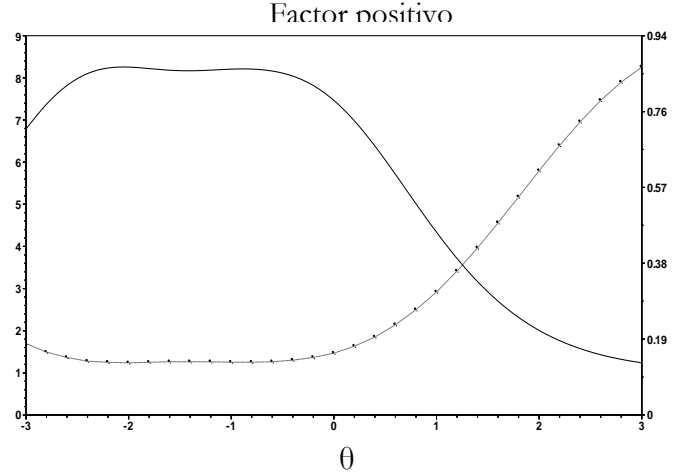

Figura 1. Funciones de Información de las dimensiones del CTI-

Tabla 6. Parámetros de discriminación y posición de los ítems del CTI-C(18) estimados mediante TRI.

Items

Negativo

1. Parece que todo me va a salir mal

2. Pienso que soy un fracaso

3. El mundo es un lugar imperfecto

4. Tengo motivos para pensar que las cosas me irán mal

5. Me odio

6. Me ocurren muchas cosas malas

7. Mi futuro es demasiado malo como para pensar en él

8. Me siento culpable por muchas cosas

9. No importa lo que haga, otras personas me ponen dificultades para conseguir lo que necesito

Positivo

1. La gente que conozco me ayuda cuando lo necesito

2. En el futuro me pasarán muchas cosas divertidas

3. La gente me quiere

4. Soy tan bueno como la gente que conozco

5. La gente importante para mí me cuida y es cariñosa conmigo

6. Solucionaré mis problemas

7. Puedo hacer bien muchas cosas

8. Soy una buena persona

9. Creo que seré feliz cuando me vaya haciendo mayor

Nota: a =discriminación; $b_{1}, b_{2}, b_{3}, b_{4}=$ parámetros de posición de las alternativas de respuesta.

$\begin{array}{llllll}a & b_{1} & b_{2} & b_{3} & b_{4} & b_{4}-b_{1}\end{array}$

$\begin{array}{cccccc}2.37 & -.17 & .6 & 1.55 & 2.08 & 2.25 \\ 3.06 & .45 & .97 & 1.62 & 2.01 & 1.56 \\ .74 & -.51 & .39 & 1.99 & 2.86 & 3.37 \\ 2.39 & -.08 & .59 & 1.53 & 1.98 & 2.06 \\ 2.78 & .71 & 1.14 & 1.79 & 2.17 & 1.46 \\ 1.89 & -.70 & .28 & 1.45 & 2.19 & 2.89 \\ 2.24 & .62 & 1.23 & 1.97 & 2.38 & 1.76 \\ 1.88 & -.56 & .26 & 1.53 & 2.13 & 2.69 \\ .91 & -.68 & .45 & 1.99 & 2.81 & 3.49\end{array}$

$\begin{array}{llllll}1.39 & -3.37 & -2.45 & -1.04 & .04 & 3.41\end{array}$

$\begin{array}{llllll}1.62 & -2.6 & -2.03 & -.55 & .34 & 2.94\end{array}$

$\begin{array}{llllll}1.7 & -2.68 & -2.13 & -.93 & .02 & 2.70\end{array}$

$\begin{array}{llllll}1.47 & -2.38 & -1.77 & -.29 & .78 & 3.16\end{array}$

$\begin{array}{llllll}1.79 & -2.60 & -2.05 & -1.13 & -.47 & 2.13\end{array}$

$\begin{array}{llllll}1.89 & -2.37 & -1.93 & -.98 & -.16 & 2.21\end{array}$

$\begin{array}{llllll}1.61 & -2.85 & -2.32 & -.94 & .23 & 3.08\end{array}$

$\begin{array}{llllll}1.55 & -3.29 & -2.72 & -1.31 & -.01 & 3.28\end{array}$

$\begin{array}{llllll}1.64 & -2.52 & -2.13 & -1.15 & -.39 & 2.13\end{array}$
Dado que los parámetros $b$ indican la dificultad de cada una de las alternativas, la diferencia entre los valores extremos $\left(b_{4}-b_{1}\right)$ señala cómo de difícil es pasar de una puntuación extrema negativa a una extrema positiva. Con finalidad terapéutica, esta diferencia, estaría señalando por qué ítems habría que comenzar a trabajar para que sea más sencillo co- 
menzar a tener éxito en la terapia. Como puede observarse en la Tabla 6 , teniendo en cuenta la diferencia entre los parámetros de posición $\left(b_{4}-b_{1}\right)$, el pensamiento negativo más sencillo de modificar sería el relativo al ítem Me odio, mientras que modificar el pensamiento No importa lo que haga, otras personas me ponen dificultades para conseguir lo que necesito, sería el más difícil de modificar.

Finalmente, para la interpretación de las puntuaciones, se muestran los baremos, en percentiles, de las dos dimensiones del CTI-C(18) en función del sexo (Tabla 7).

Tabla 7. Baremos del CTI-C(18) en función del sexo.

\begin{tabular}{lcccc}
\hline & \multicolumn{2}{c}{ Factor TP } & \multicolumn{2}{c}{ Factor TN } \\
\hline PC & Hombres & Mujeres & Hombres & Mujeres \\
5 & PD & PD & PD & PD \\
10 & $9-25$ & $9-28$ & 9 & 9 \\
20 & $26-29$ & $29-31$ & 10 & 10 \\
25 & $30-31$ & $32-34$ & 11 & 11 \\
30 & 32 & 35 & 12 & 12 \\
40 & $33-34$ & 36 & 13 & 13 \\
50 & $35-36$ & 37 & $14-15$ & 14 \\
60 & 37 & $38-39$ & $16-17$ & $15-16$ \\
70 & $38-39$ & 40 & 18 & 17 \\
75 & 40 & 41 & $19-20$ & $18-19$ \\
80 & 41 & 42 & 21 & 20 \\
90 & 42 & 43 & $22-24$ & $21-22$ \\
95 & 43 & 44 & $25-28$ & $23-26$ \\
\hline Nota: PC=Percentil; PD=Puntuación Directa. & & $27-45$ \\
\hline
\end{tabular}

$\mathrm{TN} .=$ Factor de la Tríada Negativa; $\mathrm{TP}=$ Factor de la Tríada positiva.

\section{Discusión}

La tríada cognitiva es central en el desarrollo y mantenimiento de la depresión (Beck, 1987). Por ello, contar con un instrumento en castellano, adecuadamente validado a la población infantil, que evalúe estos aspectos es importe para el trabajo preventivo y clínico con niños. De este trabajo se pueden concluir dos aspectos. El primero es que el CTI$\mathrm{C}(18)$ propuesto es un cuestionario con una adecuada validez y fiabilidad, confirmando la existencia únicamente de dos factores. En segundo lugar, se detectan diferencias estadísticamente significativas únicamente en función del sexo en las respuestas a ambas dimensiones, positiva y negativa.

La forma breve del CTI-C aquí propuesta consta de 18 ítems que conservan la misma estructura de la versión completa. Es decir, mantiene los tres ámbitos del yo, mundo y futuro y, en cada uno de ellos, el mismo número de ítems redactados de forma positiva y negativa.

Los índices de ajuste obtenidos, la información aportada por cada uno de los modelos y las propiedades psicométricas de esta versión breve, ratifican la existencia de dos únicos factores de signo opuesto: tríada positiva $(\alpha=.88)$ y tríada negativa $(\alpha=.92)$, los cuales describen pensamientos positivos y negativos, respectivamente, sobre yo, mundo y futuro.

Estos resultados no confirman el modelo trifactorial de Beck que define el CTI-C (Kaslow et al., 1992), sino que avalan una solución factorial bidimensional. Esta discrepan- cia en cuanto a su dimensionalidad puede ser producto del desarrollo cognitivo propio de las edades infantiles de la muestra. Así, los resultados parecen apoyar la idea de que los pensamientos sobre el yo, mundo y futuro son tres tipos de pensamientos sobre el yo (LaGrange et al., 2008), más que tres factores discretos (Greening et al., 2005; Zauszniewski et al., 1999). En base a estos resultados, no sería necesario mantener las tres dimensiones separadas para representar la estructura latente de los pensamientos depresivos que defiende la teoría de la depresión de Beck, al menos en la infancia. En definitiva, esta estructura psicométrica parece apoyar la idea de que estas clases de pensamientos o "productos cognitivos" en los niños (tríada cognitiva positiva y negativa) se derivan de esquemas positivos y negativos respectivamente, a imagen de lo que Clark, Beck y Alford (1999) defienden en adultos.

También es interesante señalar que estos factores muestran un patrón consistente de correlaciones. Así, los pensamientos de la tríada positiva muestran unas correlaciones sistemáticamente negativas (entre -.29 y -.41) con las puntuaciones obtenidas en depresión, ansiedad, inutilidad, autovaloración e irritabilidad, mientras que las correlaciones entre estas dificultades y los pensamientos de la tríada negativa son todas elevadas y positivas (entre .62 y .79); además, la correlación entre los dos factores es -.54, en la misma línea de la obtenida por LaGrange et al. (2008). De este análisis correlacional dos son los aspectos que se pueden considerar.

En primer lugar, la activación de un determinado tipo de pensamiento positivo o negativo podría inhibir, desactivar o mediatizar el complementario. Las implicaciones de este dato son muy importantes, tanto desde el punto de vista de la prevención como desde la perspectiva clínica. La prevención de estos trastornos psicológicos podría favorecerse tanto a nivel escolar como familiar. Un medio ambiente reforzador de las conductas infantiles, donde prime la potenciación de los aspectos positivos más que el marcaje de los negativos, inhibiría la aparición de pensamientos negativos, favoreciendo una actitud positiva ante la vida, que ejercería un efecto protector ante los trastornos psicológicos. Desde el punto de vista clínico, ante la primacía de pensamientos negativos en los niños, la estrategia terapéutica podría ser doble: centrarse en potenciar y ampliar la gama de pensamientos positivos sobre el yo, mundo y futuro del niño, y reestructurar cognitiva y conductualmente sus pensamientos negativos. En este ámbito ayudaría utilizar los parámetros de posición de los ítems (Tabla 6) para optimizar los esfuerzos terapéuticos, comenzando desde los ítems más fáciles de cambiar ("me odio") a los más difíciles.

En segundo lugar, se puede afirmar, al menos inicialmente, que la presencia de la tríada negativa favorece la aparición de sintomatología depresiva, en la línea de lo señalado entre otros autores por Greening et al. (2005). Estos autores informan que el CTI-C predice las posteriores puntuaciones en depresión valoradas con el Inventario de Depresión Infantil (CDI; Kovacs, 1992). Es evidente que esta conclusión 
ha de venir refrendada por estudios psicométricos específicamente diseñados para tal fin.

Con respecto al comportamiento diferencial en función del sexo, se observa que los niños tienen significativamente más pensamientos negativos que las niñas, pudiendo ser las explicaciones de estos resultados múltiples. Así, una pauta de crianza específica caracterizada por mayores niveles de exigencia y de crítica a los niños y a sus trabajos que a las niñas podría favorecer que los niños se juzgasen como menos capaces, valorasen el entorno como poco reforzante y más crítico, y el futuro más difícil (Gamble y Roberts, 2005). No obstante, el tamaño del efecto hallado, tanto en función de la edad como del sexo, se puede considerar bajo, por lo que el efecto de ambas variables en la valoración cognitiva de los niños parece ser reducido.

\section{Referencias}

Anderson, K. W. y Skidmore, J. R. (1995). Empirical analyses of factors in depressive cognition: The cognitive triad inventory. Journal of Clinical Psychology, 51, 603-609.

Auerbach, R. P., Eberhart, N.K., \& Abela, J. R. Z. (2010). Cognitive vulnerability to depression in Canadian and Chinese adolescents. Journal of $A b$ normal Child Psychology, 38, 57-68.

Beck, A. T. (1967). Depression: Clinical, experimental, and theoretical aspects. New York: Harper y Row.

Beck, A. T. (1983). Cognitive therapy of depression: New perspectives. In P. J. Clayton y J. E. Barrett (Eds.), Treatment of depression: Old controversies and new approaches (pp. 265-290). New York: Raven Press.

Beck, A. T. (1987). Cognitive models of depression. Journal of Cognitive Pychotherapy, 1, 5-37.

Beck, A. T. (1991). Cognitive Therapy. A 30-Years Retrospective. American Psychologist, 46(4), 368-375.

Beck, A. T., Rush, A., Shaw, B. y Emery, G. (1979). Cognitive Therapy of Depression. John Wiley y Sons: Chichester. 1979.

Beckham, E. E., Leber, W. R., Watkins, J. T., Boyer, J. L. y Cook, J. B. (1986). Development of an instrument to measure Beck's cognitive triad: The Cognitive Triad Inventory. Journal of Consulting and Clinical Psychology, 54, 566-567.

Bernaras, E., Jaureguizar, J., Soroa, M., Ibabe, I. y De las Cuevas, C. (2013). Evaluación de la sintomatología depresiva en el contexto escolar y variables asociadas. Anales de Psicología, 29(1), 131-140.

Beshai, S. (2010). Cognition and depression in egypt and canada: An examination of the cognitive triad. (Order No. MR69393, University of Calgary (Canada)). ProQuest Dissertations and Theses, 65. Retrieved from http:// search.proquest.com/docview/847250047?accountid=14542. (847250047).

Chang, H-J., Lin, M-F. y Lin, K-H. (2007). The mediating and moderating roles of the cognitive triad on adolescent suicidal ideation. Nursing Research, 56(4), 252-259.

Clark, D. A., Beck, A. T., y Alford, B. A. (1999). Scientific foundations of cognitive theory and therapy of depression. New York: Wile.

Cliff, N. (1993). Dominance statistics: Ordinal Analyses to Answer Ordinal Questions. Psychological Bulletin, 114(3), 494-509.doi:10.1037/00332909.114.3.494.

Dobson, K. S. y Dozois, D. J. A. (2008). Introduction: Assessing risk and resilience factors in models of depression. En K. S. Dobson y D. J. A. Dozois (Eds.), Risk factors in depression (pp. 1-16) San Diego, CA: Academic Press. doi 10.1016/ B978-0-08-045078-0.00001-0.

Elosua, O. y Zumbo, B. O. (2008). Coeficientes de fiabilidad para escalas de respuesta categórica ordenada. Psicothema, 20(4), 896-901.

Estévez, A. y Calvete, E. (2009). Mediación a través de pensamientos automáticos de la relación entre esquema y síntomas de depresión. Anales de Psicología, 25(1), 27-35.
Dedicar la investigación a dilucidar estos aspectos es una tarea importante, dada la proyección que sobre la salud en la infancia tendría conocer las variables que se encuentran en la base de tales dificultades. Así, se podrían elaborar programas preventivos y terapéuticos adecuados en pos del equilibrio emocional de la infancia.

Como ocurre con cualquier trabajo, también en este aparecen ciertas limitaciones que han de tenerse en cuenta. Los presentes resultados se han obtenido con una muestra de población general, donde se ha valorado la presencia de sintomatología depresiva o ansiosa y no un trastorno, por lo que quizás no sean representativos de los pensamientos depresivos o ansiosos de los niños diagnosticados con un trastorno depresivo mayor. Por tanto, este estudio debería repetirse con una población clínica y comprobar si esta estructura factorial sigue siendo representativa en estas condiciones.

Gamble, S. y Roberts, J. (2005). Adolescents' perceptions of primary caregivers cognitive style: The roles of attachment security and gender. $\operatorname{Cog}$ nitive Therapy and Research, 29, 123-141.

Greening, L., Stoppelbein, L., Dhossche, D. y Martin, W. (2005). Psychometric evaluation of a measure of Beck's negative cognitive triad for youth: Applications for African-American and Caucasian youth. Depression and Anxiety, 21, 161-169.

Gomez-Benito, J., Hidalgo, M.D., \& Zumbo, B.D. (2013). Effectiveness of Combining Statistical Tests and Effect Sizes When Using Logistic Discriminant Function Regression to Detect Differential Item Functioning for Polytomous Items. Educational and Psychological Measurement, 73(5), 875-897. doi:10.1177/0013164413492419.

Haaga, D. A. F., Dyck, M.J. y Ernst, D. (1991). Empirical status of cognitive teory of depression. Psychological Bulletin, 110, 215-236.

Kaslow, N. J., Stark, K.D., Printz, B., Livingston, R. y Tsai, S.L. (1992). Cognitive triad inventory for children: Development and relation to depression and anxiety. Journal of Clinical Child Psychology, 21, 339-347.

Kovacs, M. (1992). Children's Depression Inventory. Toronto: Multi-Health Systems.

LaGrange, B., Cole, D. A., Dallaire, D. H., Ciesla, J. A., Pineda, A. Q., Truss, A. E. y Folmer, A. (2008). Developmental changes in depressive cognitions: A longitudinal evaluation of the Cognitive Triad Inventory for children. Psychological Assessment, 20(3), 217-226.

Lozano, L.M., García-Cueto E. y Muñiz, J. (2008). Effect of the number of response categories on the reliability and validity of rating scales. Methodology, 4, 73-79.

Lozano, L., García-Cueto, E. y Lozano, L. M. (2010). Cuestionario EducativoClínico: Ansiedad y Depresión (CECAD). TEA Ediciones: Madrid.

Lozano, L., García-Cueto, E., Martín, M. y Lozano, L. M. (2012). Desarrollo y validación del Inventario de Perfeccionismo Infantil (I.P.I.). Psicotbema, 24(1), 149-155.

Lozano, L.M., García-Cueto E. y Muñiz, J. (2008). Effect of the number of response categories on the reliability and validity of rating scales. Methodology, 4, 73-79.

McIntosh, C. N. y Fisher, D. G. (2000). Beck's cognitive triad: One versus three factors. Canadian Journal of Behavioural Science, 32, 153-157.

Morse, D.T. (1999). Minsize2: a Computer Program for Determining Effect Size and Minimum Sample Size for Statistical Significance for Univariate, Multivariate, and Nonparametric Tests. Educational and Psychological Measurement, 59, 518-531.doi:10.1177/00131649921969901.

Muñiz, J., Elosua, P. y Hambleton, R. K. (2013). International Test Commission Guidelines for test translation and adaptation: Second edition. Psicothema, 25(2), 151-157.

Muñiz, J., Fidalgo, A.M., García-Cueto, E. Martínez, R. y Moreno, R. (2005). Análisis de items. Madrid: La Muralla. 
Muñiz, J., García-Cueto, E. y Lozano, L.M. (2005). Item format and the psychometric properties of the Eysenck Personality Questionnaire. Personality and Individual Differences, 38(1), 61-69.

Morris, M. C., Ciesla, J. A. y Garber, J. (2008). A prospective study of the cognitive-stress model of depressive symptoms in adolescents. Journal of Abnormal Psychology, 117(4), 719-734.

Reinherz, H. Z., Tanner, J. L., Berger, S. R., Beardslee, W. R. y Fitzmaurice, G. M. (2006). Adolescent suicidal ideation as predictive of psychopathology, suicidal behavior, and compromised functioning at age 30 . American Journal of Psychiatry, 163, 1226-1232.

Samejima, F. (2004). Graded response model. En K. Kempf-Leonard (Ed.), Encyclopedia of Social Measurement. New York: Academic Press.
Sanchez, A. y Vázquez, C. (2012). Sesgos de Atención Selectiva como Factor de Mantenimiento y Vulnerabilidad a la Depresión: Una Revisión Crítica. Terapia Psicológica, 30(3), 103-117.

Shin, W. S. (2008). The Relations of Cognitive Triad, Dysfunctional Attitudes, Automatic Thoughts, and Irrational Beliefs with Test Anxiety. Current Psychology, 27, 177-191.

Zauszniewski, J. A., Panitrat, R. y Youngblut, J. M. (1999). The Children's Cognitive Triad Inventory: Reliability, validity, and congruence with Beck's cognitive theory of depression. Journal of Nursing Measurement, 7(2), 101-115.

(Artículo recibido: 15-01-2014; revisado: 10-09-2014; aceptado: 26-09-2014) 


\section{Anexo I. Inventario de la Tríada Cognitiva para Niños, CTI-C(18)}

Nombre y Apellidos: Fecha de Nacimiento: Fecha de hoy:
Sexo: Hombre Mujer (Rodea lo que seas) Edad: Curso:

A continuación hay una lista de cosas que ocurren a las personas y que las personas piensan o sienten. Te pedimos que contestes con la máxima seriedad y sin engañar a todas las frases que aparecen, aunque sean muy personales. Las contestaciones son absolutamente secretas. Lee cuidadosamente cada frase y pon una cruz sobre Nunca, Casi Nunca, Algunas Veces, Casi Siempre o Siempre, dependiendo de cuál sea la palabra que mejor describa lo que piensas o te pasa.

No dejes ninguna frase sin contestar.

\begin{tabular}{|l|l|l|l|l|}
\hline Nunca & Casinaca & $\begin{array}{l}\text { Algunas } \\
\text { Veces }\end{array}$ & $\begin{array}{l}\text { Casi } \\
\text { Siempre }\end{array}$ & Siempre \\
\hline
\end{tabular}

\begin{tabular}{|c|c|c|c|c|c|}
\hline 1. Parece que todo me va a salir mal. & Nunca & Casi Nunca & Algunas Veces & Casi Siempre & Siempre \\
\hline 2. Pienso que soy un fracaso. & Nunca & Casi Nunca & Algunas Veces & Casi Siempre & Siempre \\
\hline 3. La gente que conozco me ayuda cuando lo necesito. & Nunca & Casi Nunca & Algunas Veces & Casi Siempre & Siempre \\
\hline 4. En el futuro me pasarán muchas cosas divertidas. & Nunca & Casi Nunca & Algunas Veces & Casi Siempre & Siempre \\
\hline 5. La gente me quiere. & Nunca & Casi Nunca & Algunas Veces & Casi Siempre & Siempre \\
\hline 6. Soy tan bueno como la gente que conozco. & Nunca & Casi Nunca & Algunas Veces & Casi Siempre & Siempre \\
\hline 7. El mundo es un lugar imperfecto & Nunca & Casi Nunca & Algunas Veces & Casi Siempre & Siempre \\
\hline 8. Tengo motivos para pensar que las cosas me irán mal. & Nunca & Casi Nunca & Algunas Veces & Casi Siempre & Siempre \\
\hline 9. La gente importante para mí me cuida y es cariñosa conmigo & Nunca & Casi Nunca & Algunas Veces & Casi Siempre & Siempre \\
\hline 10. Me odio. & Nunca & Casi Nunca & Algunas Veces & Casi Siempre & Siempre \\
\hline 11. Solucionaré mis problemas. & Nunca & Casi Nunca & Algunas Veces & Casi Siempre & Siempre \\
\hline 12. Me ocurren muchas cosas malas. & Nunca & Casi Nunca & Algunas Veces & Casi Siempre & Siempre \\
\hline 13. Puedo hacer bien muchas cosas. & Nunca & Casi Nunca & Algunas Veces & Casi Siempre & Siempre \\
\hline 14. Mi futuro es demasiado malo como para pe & Nunca & Casi Nunca & Algunas Veces & Casi Siempre & Siempre \\
\hline 15. Me siento culpable por muchas cosas. & Nunca & Casi Nunca & Algunas Veces & Casi Siempre & Siempre \\
\hline $\begin{array}{l}\text { 16. No importa lo que haga, otras personas me ponen dificulta- } \\
\text { des para conseguir lo que necesito. }\end{array}$ & Nunca & Casi Nunca & Algunas Veces & Casi Siempre & Siempre \\
\hline 17. Soy una buena persona. & Nunca & Casi Nunca & Algunas Veces & Casi Siempre & Siempre \\
\hline 18. Creo que seré feliz cuando me vaya haciendo mayor. & Nunca & Casi Nunca & Algunas Veces & Casi Siempre & Siempre \\
\hline
\end{tabular}

